\title{
CARTAN ALGEBRAS AND INVOLUTIONS
}

\author{
KARLHEINZ SPINDLER
}

(Communicated by Jonathan M. Rosenberg)

\begin{abstract}
We identify Cartan algebras in certain semidirect products and prove that every involution of a real Lie algebra leaves invariant some Cartan algebra. Moreover, we establish the adaptability of invariant Cartan algebras and invariant Levi decompositions and prove some conjugacy theorems for invariant Cartan algebras.
\end{abstract}

\section{INTRODUCTION AND NOTATION}

A Cartan algebra of a Lie algebra is a nilpotent subalgebra which is its own normalizer. The importance of Cartan algebras for the structure and representation theory of semisimple Lie algebras is well known, but Cartan algebras also play a prominent role in arbitrary Lie algebras, for example, in the Lie theory of semigroups and in the theory of causal structures on homogeneous spaces; see [3] and the bibliography included in this monograph. Although a rather complete account on Cartan algebras is available (see [1, Chapter VII]), the results presented here seem to have escaped attention until now.

An involution of a Lie algebra $\mathfrak{g}$ is an automorphism $\sigma: \mathfrak{g} \rightarrow \mathfrak{g}$ such that $\sigma^{2}=\mathrm{id}_{\mathfrak{g}}$; involutions arise naturally in the study of symmetric spaces. Writing $L:=\{x \in \mathfrak{g} \mid \sigma x=x\}$ and $Q:=\{x \in \mathfrak{g} \mid \sigma x=-x\}$, we obtain the decomposition $\mathfrak{g}=L \oplus Q$ which is symmetric in the following sense:

$$
[L, L] \subseteq L, \quad[L, Q] \subseteq Q, \quad[Q, Q] \subseteq L .
$$

Conversely, every symmetric decomposition of a Lie algebra uniquely determines an involution. Two symmetric decompositions $\mathfrak{g}=L_{1} \oplus Q_{1}=L_{2} \oplus Q_{2}$ are called compatible if

$$
\mathfrak{g}=\left(L_{1} \cap L_{2}\right) \oplus\left(L_{1} \cap Q_{2}\right) \oplus\left(Q_{1} \cap L_{2}\right) \oplus\left(Q_{1} \cap Q_{2}\right) .
$$

It is easy to check that this holds if and only if $\sigma_{1} \sigma_{2}=\sigma_{2} \sigma_{1}$ for the corresponding involutions.

The intersection over the descending central series of a Lie algebra $\mathfrak{g}$ is denoted by $C^{\infty} \mathfrak{g}$. If $X$ and $Y$ are arbitrary subsets of a Lie algebra $\mathfrak{g}$, we denote the centralizer and the normalizer of $Y$ in $X$ by

$$
Z_{X} Y:=\{x \in X \mid[x, Y]=\{0\}\} \quad \text { and } N_{X} Y:=\{x \in X \mid[x, Y] \subseteq Y\} .
$$

Received by the editors November 12, 1990 and, in revised form, April 15, 1991 and June 1, 1992.

1991 Mathematics Subject Classification. Primary 17B05. 
If $X$ is a subalgebra of $\mathfrak{g}$, then so is $Z_{X} Y$; if $X$ is a subalgebra of $\mathfrak{g}$ and $Y$ is a vector space, then $N_{X} Y$ is a subalgebra of $\mathfrak{g}$. Moreover, the set of regular elements of $\mathfrak{g}$ is denoted by $\operatorname{Reg}(\mathfrak{g})$.

For a representation $\phi: \mathfrak{g} \rightarrow \operatorname{gl}(V)$ of a Lie algebra $\mathfrak{g}$ we let

$$
V^{0}(\mathfrak{g}):=\left\{v \in V \mid \text { for any } x \in \mathfrak{g} \text { there is an } n \in \mathbb{N} \text { such that } \phi(x)^{n} v=0\right\}
$$

and

$$
V_{0}(\mathfrak{g}):=\{v \in V \mid \phi(x) v=0 \text { for every } x \in L\} .
$$

Trivially, $V_{0}(\mathfrak{g})$ is a $\mathfrak{g}$-submodule of $V$. The identity

$$
\phi(x)^{n} \phi(y)=\sum_{k=0}^{n}\left(\begin{array}{l}
n \\
k
\end{array}\right) \phi\left((\operatorname{ad} x)^{k} y\right) \phi(x)^{n-k} \quad(x, y \in H)
$$

shows that also $V^{0}(\mathfrak{g})$ is a $\mathfrak{g}$-submodule of $V$ if $\mathfrak{g}$ is nilpotent. Clearly, $V_{0}(\mathfrak{g}) \subseteq$ $V^{0}(\mathfrak{g})$, and $V_{0}(\mathfrak{g})=\{0\}$ if and only if $V^{0}(\mathfrak{g})=\{0\}$; in this case, the action of $\mathfrak{g}$ on $V$ is called effective.

Let $A$ and $B$ be two Lie algebras and $\phi: A \rightarrow \operatorname{Der} B$ a Lie algebra homomorphism. Then the semidirect product of $A$ and $B$ with respect to $\phi$ is denoted by $\mathfrak{g}:=A \ltimes_{\phi} B$; i.e., $\mathfrak{g}=A \times B$ with the Lie bracket

$$
\left[\left(\begin{array}{l}
a \\
b
\end{array}\right),\left(\begin{array}{l}
a^{\prime} \\
b^{\prime}
\end{array}\right)\right]=\left(\begin{array}{c}
{\left[a, a^{\prime}\right]} \\
\phi(a) b^{\prime}-\phi\left(a^{\prime}\right) b+\left[b, b^{\prime}\right]
\end{array}\right) .
$$

\section{THE RESULTS}

The first lemma generalizes Problem VII.2(7) in [1, p. 58] and is of some independent interest. Since many examples are constructed as semidirect products, it gives a convenient criterion for identifying a Cartan algebra. The base-field is arbitrary in this lemma.

Lemma 1. Suppose that a Lie algebra $L$ acts on a nilpotent Lie algebra $N$ via $\phi: L \rightarrow \operatorname{Der} N$. If $H$ is a Cartan algebra of $L$, then $\mathfrak{h}:=H \ltimes_{\phi} V^{0}(H)$ is a Cartan algebra of $\mathfrak{g}:=L \ltimes_{\phi} N$.

Proof. (a) Let $V:=N^{0}(H)$. The identity

$$
\phi(x)^{n}[a, b]=\sum_{k=0}^{n}\left(\begin{array}{l}
n \\
k
\end{array}\right)\left[\phi(x)^{k} a, \phi(x)^{n-k} b\right] \quad(x \in H)
$$

shows that $[V, V] \subseteq V$ so that $V$ is a (necessarily nilpotent) subalgebra of $N$. Also, $V$ is an $H$-submodule of $N$. This shows that $\mathfrak{h}$ is a well-defined subalgebra of $\mathfrak{g}$.

(b) We prove that $\mathfrak{h}$ is nilpotent. We are in the situation that $V$ and $H$ are nilpotent algebras and $\phi: H \rightarrow \operatorname{Der} V$ is a homomorphism such that all operators $\phi(h)(h \in H)$ are nilpotent. We show by induction on $n:=\operatorname{dim} H \ltimes_{\phi}$ $V$ that in this situation the algebra $H \ltimes_{\phi} V$ is always nilpotent.

The case $n=0$ is trivial. Let $n \geq 1$. If $V=\{0\}$ then $H \ltimes_{\phi} V \cong H$, and we are done. Otherwise the center $Z(V)$ of $V$ is nonzero (because $V$ is nilpotent) and invariant under the action of $H$. By Engel's theorem, there is an element $v_{0} \neq 0$ in $Z(V)$ with $\phi(H) v_{0}=\{0\}$. Now it is easy to check that $\left(v_{0}, 0\right)$ belongs to the center $Z(\mathfrak{h})$ of $\mathfrak{h}$, whence $Z(\mathfrak{h}) \cap V \neq\{0\}$ so 
that $\mathfrak{h} /(Z(\mathfrak{h}) \cap V)=H \ltimes_{\phi}(V /(Z(\mathfrak{h}) \cap V))$ has lower dimension than $\mathfrak{h}$ but is again of the type under consideration. By induction hypothesis, $\mathfrak{h} /(Z(\mathfrak{h}) \cap V)$ is nilpotent. But then so is $\mathfrak{h}$, as a central extension of $\mathfrak{h} /(Z(\mathfrak{h}) \cap V)$.

(c) We show that $\mathfrak{h}$ is its own normalizer in $\mathfrak{g}$. Let $(x, n) \in N_{\mathfrak{g}} \mathfrak{h}$ so that

$$
\text { ( } \left.\left[x, x^{\prime}\right], \phi(x) v^{\prime}-\phi\left(x^{\prime}\right) n+\left[n, v^{\prime}\right]\right) \in H \times V \quad \text { for all }\left(x^{\prime}, v^{\prime}\right) \in H \times V \text {. }
$$

Then $x \in N_{L} H=H$. Now choose $\left(x^{\prime}, v^{\prime}\right):=(h, 0)$ to see that $\phi(h) n \in V$ for all $h \in H$. But then the definition of $V$ implies that $n \in V$. This shows $(x, n) \in H \times V=\mathfrak{h}$, so the condition $N_{\mathfrak{g}} \mathfrak{h}=\mathfrak{h}$ is established.

The second lemma has been singled out from the proof of Theorem 1 to avoid an interruption of the chain of reasoning; it will also be needed in the proof of Theorem 3. The base-field $K$ is again arbitrary except for the conjugacy claim in part (b) where char $K=0$ is assumed.

Lemma 2. Suppose the nilpotent Lie algebra $H$ acts effectively on a vector space $V$ via $\phi: H \rightarrow \operatorname{gl}(V)$. Consider the semidirect product $\mathfrak{g}:=H \ltimes_{\phi} V$.

(a) The involutions of $\mathfrak{g}$ which leave $V$ invariant are exactly the mappings

$$
\sigma\left(\begin{array}{l}
h \\
v
\end{array}\right)=\left(\begin{array}{c}
A h \\
\phi(A h)(D-\mathbf{1}) u+D v
\end{array}\right)
$$

where $A: H \rightarrow H$ and $D: V \rightarrow V$ are involutions with $D \circ \phi(h) \equiv \phi(A h) \circ D$ and $u$ is a vector in $V$.

(b) Given such an involution $\sigma$, there is a Cartan algebra of $\mathfrak{g}$ which is invariant under $\sigma$. Moreover, if $H_{1}$ and $H_{2}$ are two $\sigma$-invariant Cartan algebras of $\mathfrak{g}$, there is a vector $v \in V$ such that $\sigma\left(\begin{array}{l}0 \\ v\end{array}\right)=\left(\begin{array}{l}0 \\ v\end{array}\right)$ and $H_{2}=e^{\text {ad }\left(\begin{array}{l}0 \\ v\end{array}\right)} H_{1}$.

Proof. (a) Since $\sigma: \mathfrak{g} \rightarrow \mathfrak{g}$ is a linear mapping which leaves $V$ invariant, we can represent it in block form $\sigma=\left(\begin{array}{ll}A & 0 \\ C & D\end{array}\right)$ with respect to the decomposition $\mathfrak{g}=H \oplus V$. A straightforward calculation shows that $\sigma$ is an involution of $\mathfrak{g}$ if and only if $A: H \rightarrow H$ and $D: V \rightarrow V$ are involutions such that the following conditions hold:

(1) $D \circ \phi(h) \equiv \phi(A h) \circ D$ (which is equivalent to $\phi(h) \circ D \equiv D \circ \phi(A h))$;

(2) $C\left(\left[h, h^{\prime}\right]\right) \equiv \phi(A h) C\left(h^{\prime}\right)-\phi\left(A h^{\prime}\right) C(h)$;

(3) $C A+D C=0$.

Let us show that every mapping $C: H \rightarrow V$ which satisfies condition (2) is of the form

$$
C(h)=\phi(A h) w \quad \text { for some } w \in V .
$$

Consider $V_{1}:=V \times K$ as an $H$-module via

$$
h *\left(\begin{array}{l}
v \\
\lambda
\end{array}\right):=\left(\begin{array}{c}
\phi(A h) v-\lambda C(h) \\
0
\end{array}\right)
$$

and let $V_{2}:=K$ be the trivial one-dimensional $H$-module. Then

$$
\begin{aligned}
& V_{1} \rightarrow V_{2} \\
& f:\left(\begin{array}{l}
v \\
\lambda
\end{array}\right) \mapsto \lambda
\end{aligned}
$$

is a surjective homomorphism of $H$-modules. Hence $f\left(V_{1}^{0}(H)\right)=V_{2}^{0}(H)=$ $K \ni 1$ (see [1, VII.1.3, Proposition 9(iv), p. 14]). Thus there is an element 
$w \in V$ with $(w, 1) \in V_{1}^{0}(H)$. Now $V_{1}^{0}(H) \cap(V \oplus\{0\})=V^{0}(H)=\{0\}$ by assumption. This means that $V_{1}^{0}(H)$ intersects a hyperplane in $\mathfrak{g}$ trivially and, hence, must be one-dimensional and therefore coincide with $\left(V_{1}\right)_{0}(H)$. This gives $\left(V_{1}\right)_{0}(H)=K(w, 1)$. Hence for any $h \in H$ the equation

$$
\left(\begin{array}{l}
0 \\
0
\end{array}\right)=h *\left(\begin{array}{c}
w \\
1
\end{array}\right)=\left(\begin{array}{c}
\phi(A h) w-C(h) \\
0
\end{array}\right)
$$

holds, which shows that $C(h) \equiv \phi(A h) w$.

With this explicit form for $C$, condition (3) reads $\phi(h) w \equiv-D \circ \phi(A h) w \equiv$ $-\phi(h) D w$; i.e., $\phi(h)(D+\mathbf{1}) w=0$ for all $h \in H$. Since the action of $H$ is effective, this gives $w \in \operatorname{ker}(D+1)=\operatorname{im}(D-1)$, and the claim is established.

(b) Note that $H=H \oplus\{0\}$ is a Cartan algebra of $\mathfrak{g}$ by Lemma 1. Since $\mathfrak{g}$ is solvable, every other Cartan algebra of $\mathfrak{g}$ is of the form $e^{\operatorname{ad} x} H$ for some element $x \in C^{\infty} \mathfrak{g} \subseteq\{0\} \times V$ if we assume zero characteristic (see [1, VII.3.4, Theorem 3, p. 31]). $\nmid$ Now

$$
H_{v}:=e^{\text {ad }\left(\begin{array}{l}
0 \\
v
\end{array}\right)} H=\left\{\left(\begin{array}{c}
h \\
-\phi(h) v
\end{array}\right) \mid h \in H\right\}
$$

observe that

$$
H_{v_{2}}=e^{\operatorname{ad}\left(v_{2}-v_{1}\right)} H_{v_{1}} \quad \text { for all } v_{1}, v_{2} \in V .
$$

Let $\sigma$ be given as in part (a). It is easy to check that $H_{v}$ is $\sigma$-invariant if and only if $v \in u+\operatorname{ker}(D-1)$. Hence if $H_{v_{1}}$ and $H_{v_{2}}$ are $\sigma$-invariant, then $v_{2}-v_{1} \in \operatorname{ker}(D-1)$. On the other hand, an element $\left(\begin{array}{l}0 \\ v\end{array}\right)$ is a fixed point of $\sigma$ if and only if $v \in \operatorname{ker}(D-\mathbf{1})$. This gives the claim.

Theorem 1. Let $\mathfrak{g}$ be a finite-dimensional real Lie algebra and $\sigma: \mathfrak{g} \rightarrow \mathfrak{g}$ an involution. Then there is a Cartan algebra $H$ which is invariant under $\sigma$.

Proof. Suppose the claim is false. Then there is a counterexample $\mathfrak{g}$ of minimal dimension. Clearly, $\mathfrak{g}$ cannot be nilpotent because otherwise $\mathfrak{g}$ itself would be the only Cartan algebra of $\mathfrak{g}$.

(a) Suppose first that $\mathfrak{g}$ is semisimple. Let $\mathfrak{g}=L \oplus Q$ be the symmetric decomposition associated with $\sigma$. There is a Cartan decomposition $\mathfrak{g}=K \oplus P$ which is compatible with this decomposition; i.e.,

$$
\mathfrak{g}=(L \cap K) \oplus(L \cap P) \oplus(Q \cap K) \oplus(Q \cap P)
$$

(see [2, Chapter III, Exercise B.4, p. 192] or [5, Proposition 7.1.1, p. 114]). We shall construct a Cartan algebra $H$ which is adapted to this decomposition. Pick any maximal abelian subspace $A_{P}$ in $Q \cap P$, and let $A_{K}$ be maximal abelian in $Z_{Q \cap K}\left(A_{P}\right)$. It is easy to check that $A:=A_{K}+A_{P}$ is maximal abelian in $Q$ and that $Z_{L} A=\left(Z_{L} A\right) \cap K+\left(Z_{L} A\right) \cap P$. Now pick $B_{P}$ maximal abelian in $\left(Z_{L} A\right) \cap P$, and let $B_{K}$ be maximal abelian in $Z_{\left(Z_{L} A\right) \cap K}\left(B_{P}\right)$. As before, we easily verify that $B:=B_{K}+B_{P}$ is maximal abelian in $Z_{L} A$; moreover, $H:=B+A$ is maximal abelian in $\mathfrak{g}$. We claim that the operator $\operatorname{ad}(h)$ is semisimple for every $h \in H$. Indeed, write $h=b_{K}+b_{P}+a_{K}+a_{P}$. Each of the four operators $\operatorname{ad}\left(b_{K}\right), \operatorname{ad}\left(b_{P}\right), \operatorname{ad}\left(a_{K}\right), \operatorname{ad}\left(a_{P}\right)$ is semisimple. I† $\operatorname{But}$

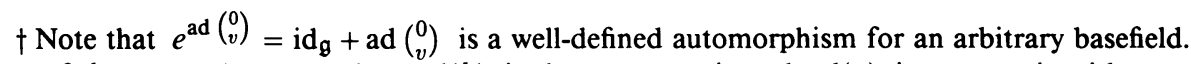

t† If $k \in K$ and $p \in P$, then $\operatorname{ad}(k)$ is skew-symmetric and $\operatorname{ad}(p)$ is symmetric with respect to the inner product $-B(\cdot, \theta \cdot)$; in particular, $\operatorname{ad}(k)$ and $\operatorname{ad}(p)$ are semisimple. 
these four operators commute, so they are simultaneously diagonalizable over $\mathbb{C}$. Hence $\operatorname{ad}(h)=\operatorname{ad}\left(b_{K}\right)+\operatorname{ad}\left(b_{P}\right)+\operatorname{ad}\left(a_{K}\right)+\operatorname{ad}\left(a_{P}\right)$ is semisimple. Now a subalgebra $H$ of a semisimple Lie algebra is a Cartan algebra if and only if $H$ is maximal abelian and all operators $\operatorname{ad}(h)$ are semisimple. This shows that $H$ is a Cartan algebra. But $H$ is obviously $\sigma$-invariant, contradicting our choice of $\mathfrak{g}$ as a counterexample. Hence $\mathfrak{g}$ cannot be semisimple.

(b) Let $R$ be the radical of $\mathfrak{g}$; then $R \neq\{0\}$ by (a). Since $R$ is a characteristic ideal of $\mathfrak{g}$, we can choose a minimal $\sigma$-invariant ideal $I \neq\{0\}$ of $\mathfrak{g}$ inside $R$. Then $I$ is solvable so that $[I, I] \subsetneq I$. On the other hand, $[I, I]$ is again a $\sigma$-invariant ideal of $\mathfrak{g}$ and, hence, must be $\{0\}$ due to the minimality of $I$. This means that $I$ is abelian. Consequently, $I \neq \mathfrak{g}$.

Now $\sigma$ induces an involution $\bar{\sigma}$ on the quotient algebra $\overline{\mathfrak{g}}:=\mathfrak{g} / I$ which has lower dimension than $\mathfrak{g}$. Since $\mathfrak{g}$ was a minimal counterexample, there is a $\bar{\sigma}$ invariant Cartan algebra $\bar{H}$ of $\overline{\mathfrak{g}}$. But $\bar{H}$ is necessarily of the form $(H+I) / I$ where $H$ is a Cartan algebra of $\mathfrak{g}$; see [1, VII.2.3, Corollary 2, p. 24]. The $\bar{\sigma}$-invariance of $\bar{H}$ yields $\sigma(H) \subseteq H+I$. Now $U:=H+I$ is a subalgebra of $\mathfrak{g}$ because $I$ is abelian, and $\sigma$ leaves $U$ invariant so that $\left.\sigma\right|_{U}$ is an involution of $U$. Suppose $U \subsetneq \mathfrak{g}$. Then $U$ possesses a $\sigma$-invariant Cartan algebra $\tilde{H}$ because $\mathfrak{g}$ is a minimal counterexample. But $\widetilde{H}$ is necessarily a Cartan algebra of $\mathfrak{g}$ because $U$ contains the Cartan algebra $H$ of $\mathfrak{g}$ so that $\operatorname{rank} U=\operatorname{rank} \mathfrak{g}$. But we started with the assumption that $\mathfrak{g}$ has no $\sigma$-invariant Cartan algebra! This shows that $U=\mathfrak{g}$.

(c) We are left with the case that $\mathfrak{g}=H+I$ where $I$ is abelian and a minimal $\sigma$-invariant ideal. Observe that $H \cap I$ is an ideal of $\mathfrak{g}$ because

$$
[\mathfrak{g}, H \cap I]=[H+I, H \cap I]=[H, H \cap I] \subseteq H \cap I .
$$

Also, $H \cap I$ is properly contained in $I$ because otherwise $I \subseteq H$ so that $\mathfrak{g}=H$ would be nilpotent and hence its only Cartan algebra, which is absurd by the choice of $\mathfrak{g}$.

Let us show that the sum $\mathfrak{g}=H+I$ is direct. Suppose $H \cap I \neq\{0\}$. Then

$$
I=(H \cap I) \oplus \sigma(H \cap I) .
$$

Indeed, $(H \cap I)+\sigma(H \cap I) \subseteq I$ is a nonzero $\sigma$-invariant ideal of $\mathfrak{g}$ and, hence, is $I$ by the minimality of $I$. The sum must be direct because the intersection of $H \cap I$ and $\sigma(H \cap I)$ is a $\sigma$-invariant ideal properly contained in $I$ and, hence, must be zero. We want to establish that

$$
\mathfrak{g}=\underbrace{(H \cap \sigma H) \oplus \overbrace{(H \cap I)}^{(H \sigma(H \cap I)}}_{H} .
$$

Since $I=(H \cap I) \oplus \sigma(H \cap I)$, we have $\mathfrak{g}=H+I=H+\sigma(H \cap I)$. But this is a direct sum because $H \cap \sigma(H \cap I)=(H \cap I) \cap \sigma(H \cap I)=\{0\}$. Hence $\mathfrak{g}=H \oplus \sigma(H \cap I)$. Also, $(H \cap \sigma H) \cap(H \cap I)=(H \cap I) \cap(\sigma H \cap I)=$ $(H \cap I) \cap \sigma(H \cap I)=\{0\}$. So the remaining condition $H=(H \cap \sigma H) \oplus(H \cap I)$ is a consequence of the following dimension argument:

$$
\begin{aligned}
& \operatorname{dim}(H \cap \sigma H)+\operatorname{dim}(H \cap I)=2 \operatorname{dim} H-\operatorname{dim} \overbrace{(H+\sigma H)}^{=\mathfrak{g}}+\operatorname{dim}(H \cap I) \\
& \quad=2 \operatorname{dim} H-(\operatorname{dim} \mathfrak{g}-\operatorname{dim} \sigma(H \cap I))=2 \operatorname{dim} H-\operatorname{dim} H=\operatorname{dim} H .
\end{aligned}
$$


Now from $(* *)$ we conclude by induction on $n$ that

$$
\mathfrak{g}^{(n)} \subseteq(H \cap \sigma H)^{(n)}+\left(H^{(n)} \cap I\right)+\sigma\left(H^{(n)} \cap I\right),
$$

which implies that $\mathfrak{g}$ is nilpotent. But this is absurd.

(d) It remains to treat the case $\mathfrak{g}=H \oplus I$. Lemma 1 shows that $H \oplus I^{0}(H)$ is a Cartan algebra of $\mathfrak{g}$. But since $H$ is already a Cartan algebra of $\mathfrak{g}$, we conclude that $I^{0}(H)=\{0\}$ so that the action of $H$ on $I$ is effective. Now Lemma 2 shows that $\mathfrak{g}$ possesses a $\sigma$-invariant Cartan algebra. So finally we end up with a contradiction; the theorem is proved.

We now turn to the question of the conjugacy of $\sigma$-invariant Cartan algebras under the group $\left\langle e^{\operatorname{ad} L}\right\rangle$ where $\sigma: \mathfrak{g} \rightarrow \mathfrak{g}$ is an involution and $L$ is the fixedpoint set of $\sigma$. We need the following preparatory result which shows how $\sigma$-invariant Cartan algebras can be constructed from a certain variety in $\mathfrak{g}$.

Lemma 3. Let $\mathfrak{g}$ be an $N$-dimensional real Lie algebra, and let $\sigma: \mathfrak{g} \rightarrow \mathfrak{g}$ be an involution with the corresponding symmetric decomposition $\mathfrak{g}=L \oplus Q$. Then the variety

$$
V:=\left\{x \in \mathfrak{g} \mid \operatorname{ad}(x)^{N} \sigma(x)=0\right\}
$$

has the following properties:

(a) The $\sigma$-invariant Cartan algebras of $\mathfrak{g}$ are exactly the sets $\mathfrak{g}^{0}(x)$ where $x \in V \cap \operatorname{Reg}(\mathfrak{g})$.

(b) The variety $V$ is invariant under $\left\langle e^{\mathrm{ad} L}\right\rangle$.

(c) If $x \in V$ then

$$
T_{x} V=\left\{y \in \mathfrak{g} \mid \operatorname{ad}(x)^{N} \sigma(y)+\sum_{i+j=N-1} \operatorname{ad}(x)^{i} \operatorname{ad}(y) \operatorname{ad}(x)^{j} \sigma(x)=0\right\} .
$$

Proof. (a) The Cartan algebras of $\mathfrak{g}$ are exactly the sets $\mathfrak{g}^{0}(x)$ where $x$ is a regular element of $\mathfrak{g}$; see [1, VII.3, Theorem 2(ii)]. Since $\sigma$ preserves $\operatorname{Reg}(\mathfrak{g})$, we see that $\mathfrak{g}^{0}(x)$ is $\sigma$-invariant if and only if $\sigma x \in \mathfrak{g}^{0}(x)$ because then automatically $\mathfrak{g}^{0}(x)=\mathfrak{g}^{0}(\sigma x)=\sigma\left(\mathfrak{g}^{0}(x)\right)$. But this is tantamount to saying that $\operatorname{ad}(x)^{n} \sigma(x)=0$ for some $n \in \mathbb{N}$, which means that $x \in V$.

(b) If $g \in\left\langle e^{\text {ad } L}\right\rangle$ then $g \sigma=\sigma g$; hence if $\operatorname{ad}(x)^{N} \sigma(x)=0$ then

$$
\operatorname{ad}(g x)^{N} \sigma(g x)=\left(g \operatorname{ad}(x) g^{-1}\right)^{N} g \sigma x=g \operatorname{ad}(x)^{N} \sigma(x)=g \cdot 0=0,
$$

which means that $g x \in V$.

(c) We have $y \in T_{x} V$ if and only if $\left.\frac{d}{d t}\right|_{t=0} \operatorname{ad}(x+t y)^{N} \sigma(x+t y)=0$; now a simple computation yields the claim.

Theorem 2. Let $\mathfrak{g}$ be a finite-dimensional real semisimple Lie algebra, and let $\sigma: \mathfrak{g} \rightarrow \mathfrak{g}$ be an involution with the corresponding symmetric decomposition $\mathfrak{g}=$ $L \oplus Q$. Moreover, let $V=\left\{x \in \mathfrak{g} \mid \operatorname{ad}(x)^{N} \sigma(x)=0\right\}$ where $N$ is the dimension of $\mathfrak{g}$.

(a) Let $x \in V \cap \operatorname{Reg}(\mathfrak{g})$ so that $H:=\mathfrak{g}^{0}(x)$ is a $\sigma$-invariant Cartan algebra of $\mathfrak{g}$. Then

$$
T_{x} V=[x, L] \oplus H .
$$


(b) Let $H=\mathfrak{g}^{0}(x)$ be a $\sigma$-invariant Cartan algebra, and let $H_{*}:=H \cap$ $\operatorname{Reg}(\mathfrak{g})=\left\{y \in H \mid \mathfrak{g}^{0}(y)=H\right\}$. Then the mapping

$$
\varphi: \begin{aligned}
\left\langle e^{\mathrm{ad} L}\right\rangle \times H_{*} & \rightarrow V \\
(g, y) & \mapsto g y
\end{aligned}
$$

is open at $(\mathbf{1}, x)$.

(c) The number of conjugacy classes of $\sigma$-invariant Cartan algebras under the action of $\left\langle e^{\mathrm{ad} L}\right\rangle$ is finite. $\dagger+\dagger$

Proof. Parts (a) and (b) can be proved together. We will exploit the facts that $\mathfrak{g}^{0}(x)=\mathfrak{g}_{0}(x)$ and $H$ is abelian, which are due to the semisimplicity of $\mathfrak{g}$. Let us first show that the sum $[x, L]+H$ is direct. If $[x, y]=h \in H$ with $y \in L$, then $0=\operatorname{ad}(x) h=\operatorname{ad}(x)^{2} y$ so that $y \in \mathfrak{g}^{0}(x)=\mathfrak{g}_{0}(x)$, which means $0=[x, y]=h ;$ this shows that $[x, L] \cap H=\{0\}$.

Next observe that $\varphi^{\prime}(1, x)$ maps $L \times H$ to $T_{x} V$; since $\varphi^{\prime}(1, x)(\xi, 0)=$ $\left.\frac{d}{d t}\right|_{t=0} e^{t \text { ad } \xi} x=[\xi, x]$ for all $\xi \in L$ and $\varphi^{\prime}(\mathbf{1}, x)(0, h)=\left.\frac{d}{d t}\right|_{t=0}(x+t h)=h$ for all $h \in H$, we see that

$$
[x, L] \oplus H \subseteq \operatorname{im} \varphi^{\prime}(\mathbf{1}, x) \subseteq T_{x} V .
$$

Next, since $H$ is abelian, Lemma 3(c) takes the following simpler form

$$
\begin{aligned}
T_{x} V & =\left\{y \in \mathfrak{g} \mid \operatorname{ad}(x)^{N} \sigma(y)+\operatorname{ad}(x)^{N-1} \operatorname{ad}(y) \sigma(x)=0\right\} \\
& =\left\{y \in \mathfrak{g} \mid[x, \sigma y]+[y, \sigma x] \in \operatorname{ker} \operatorname{ad}(x)^{N-1}\right\} \\
& =\{y \in \mathfrak{g} \mid[x, \sigma y]+[y, \sigma x] \in \operatorname{ker} \operatorname{ad}(x)\} \\
& =\{y \in \mathfrak{g} \mid[x,[x, \sigma y]]-[x,[\sigma x, y]]=0\} \\
& =\operatorname{ker}\left(\operatorname{ad}(x)^{2} \sigma-\operatorname{ad}(x) \operatorname{ad}(\sigma x)\right) \\
& =\operatorname{ker}\left(\operatorname{ad}(x)^{2} \sigma-\operatorname{ad}(x) \sigma \operatorname{ad}(x) \sigma\right) \\
& =\operatorname{ker}(\operatorname{ad}(x)(\mathbf{1}-\sigma) \operatorname{ad}(x) \sigma)
\end{aligned}
$$

Hence if $y \in T_{x} V$ then

$$
\begin{aligned}
\operatorname{ad}(x)^{2} \sigma(y) & =\operatorname{ad}(x) \underbrace{\left(\frac{1+\sigma}{2}+\frac{1-\sigma}{2}\right) \operatorname{ad}(x) \sigma(y)}_{\in \operatorname{im}(1+\sigma)=L} \\
& =\operatorname{ad}(x) \underbrace{\left(\frac{1+\sigma}{2}\right) \operatorname{ad}(x) \sigma(y)} \in[x, L] .
\end{aligned}
$$

Thus the linear mapping $\theta:=\left.\operatorname{ad}(x)^{2} \sigma\right|_{T_{x} V}: T_{x} V \rightarrow \mathfrak{g}$ satisfies $\operatorname{im} \theta \subseteq[x, L]$. On the other hand we have $y \in \operatorname{ker} \theta$ if and only if $\sigma(y) \in \operatorname{kerad}(x)^{2}=$ $\operatorname{ker} \operatorname{ad}(x)=H$, which implies that $y \in H$; hence $\operatorname{ker} \theta=H$. Consequently,

(2) $\operatorname{dim} T_{x} V=\operatorname{dimim} \theta+\operatorname{dim} \operatorname{ker} \theta \leq \operatorname{dim}[x, L]+\operatorname{dim} H=\operatorname{dim}([x, L] \oplus H)$.

This shows that both inclusions in (1) are in fact equalities. In particular $\varphi^{\prime}(1, x)$ is surjective; hence $\varphi$ is open at $(1, x)$ by the implicit function theorem. Thus parts (a) and (b) of the theorem are proved.

$\mathrm{t \dagger \dagger}$ For the special case that $\sigma$ is a Cartan involution see [6, Proposition 1.3.1.1-1.3.1.4, pp. 89-91]. 
To establish (c) we define an equivalence relation on $V \cap \operatorname{Reg}(\mathfrak{g})$ by

$$
x \sim y: \Leftrightarrow \mathfrak{g}^{0}(x) \text { and } \mathfrak{g}^{0}(y) \text { are conjugate under }\left\langle e^{\mathrm{ad} L}\right\rangle .
$$

If $y$ is close to $x \in \operatorname{Reg} g$ then by part (b) we can write $y=g x^{\prime}$ where $g \in\left\langle e^{\operatorname{ad} L}\right\rangle$ and $x^{\prime} \in \mathfrak{g}^{0}(x) \cap \operatorname{Reg} \mathfrak{g}$ so that $\mathfrak{g}^{0}\left(x^{\prime}\right)=\mathfrak{g}^{0}(x)$; but then $\mathfrak{g}^{0}(y)=$ $\mathfrak{g}^{0}\left(g x^{\prime}\right)=g \cdot \mathfrak{g}^{0}\left(x^{\prime}\right)=g \cdot \mathfrak{g}^{0}(x)$. This argument shows that if $y$ is close to $x$ then $x \sim y$; hence the equivalence classes under $\sim$ are open. Since each such class is the complement of the union of the other classes, the equivalence classes are also closed and hence must be unions of connected components of $V \cap \operatorname{Reg} \mathfrak{g}$. Since this set has only a finite number of connected components, the claim follows.

For solvable Lie algebras an even better conjugacy theorem holds.

Theorem 3. Let $\mathfrak{g}$ be a finite-dimensional solvable Lie algebra over a field of characteristic zero, and let $\sigma: \mathfrak{g} \rightarrow \mathfrak{g}$ be an involution with the corresponding symmetric decomposition $\mathfrak{g}=L \oplus Q$. If $H_{1}$ and $H_{2}$ are two $\sigma$-invariant Cartan algebras of $\mathfrak{g}$, then there is an element $x \in L \cap C^{\infty} \mathfrak{g}$ such that $H_{2}=e^{\operatorname{ad} x} H_{1}$.

Proof. We proceed by a reductio ad absurdum as in the proof of Theorem 1 . Suppose the claim is false, and pick a counterexample $\mathfrak{g}$ of minimal dimension; clearly $\mathfrak{g}$ cannot be nilpotent.

As in part (b) of the proof of Theorem 1, we can choose a minimal $\sigma$ invariant ideal $I \neq\{0\}$ of $\mathfrak{g}$; then $I$ is abelian and $I \neq \mathfrak{g}$. If $H_{1}$ and $H_{2}$ are $\sigma$-invariant Cartan algebras of $\mathfrak{g}$, then $\overline{H_{1}}$ and $\overline{H_{2}}$ are $\bar{\sigma}$-invariant Cartan algebras of $\overline{\mathfrak{g}}:=\mathfrak{g} / I$ where $\bar{\sigma}$ is the involution of $\overline{\mathfrak{g}}$ induced by $\sigma$. Since $\overline{\mathfrak{g}}$ is solvable again but has lower dimension than $\mathfrak{g}$ and hence is not a counterexample, there is an element $\xi \in C^{\infty} \overline{\mathfrak{g}}$ with $\bar{\sigma} \xi=\xi$ such that

$$
\overline{H_{2}}=e^{\mathrm{ad} \xi} \overline{H_{1}} .
$$

(The same notation for different exponential functions will cause no problem.) Since $C^{\infty} \overline{\mathfrak{g}}=C^{\infty} \mathfrak{g}+I$, we can write $\xi=a+I$ with $a \in C^{\infty} \mathfrak{g}$. Then also $\xi=\bar{\sigma} \xi=\sigma a+I$, and hence $\xi=\frac{1}{2}(a+\sigma a)+I$. Thus we can write $\xi=y+I$ where $y:=\frac{1}{2}(a+\sigma a)$ lies in $L \cap C^{\infty} \mathfrak{g}$ because $C^{\infty} \mathfrak{g}$ is a characteristic ideal and hence $\sigma$-invariant. Then equation (3) yields

$$
H_{2}+I \supseteq e^{\operatorname{ad} y} H_{1}=: H_{3} \text {. }
$$

Now $U:=H_{2}+I$ is a $\sigma$-invariant subalgebra of $\mathfrak{g}$, and $H_{3}$ and $H_{2}$ are $\sigma$-invariant Cartan algebras of $U$. Suppose $U \subsetneq \mathfrak{g}$. Then $U$ is not a counterexample; hence there is an element $z \in C^{\infty} \tilde{U} \subseteq C^{\infty} \mathfrak{g}$ with $\sigma z=z$ such that

$$
H_{2}=e^{\mathrm{ad} z} H_{3}=e^{\mathrm{ad} z} e^{\mathrm{ad} y} H_{1} .
$$

Since $\left\{x \in C^{\infty} \mathfrak{g} \mid \sigma x=x\right\}$ is a nilpotent algebra, the exponential function of the group $\left\langle\left\{e^{\text {ad } x} \mid x \in C^{\infty} \mathfrak{g}, \sigma x=x\right\}\right\rangle$ is surjective. Hence we can find an element $x \in C^{\infty} \mathfrak{g}$ such that $\sigma x=x$ and $e^{\operatorname{ad} z} e^{\operatorname{ad} y}=e^{\operatorname{ad} x}$ so that $H_{2}=e^{\operatorname{ad} x} H_{1}$, contradicting our choice of $\mathfrak{g}$ as a counterexample. We conclude that $U=\mathfrak{g}$. Part (c) of the proof of Theorem 1 shows that the sum $\mathfrak{g}=\mathrm{H}_{2}+I$ must then be direct. But for an algebra of the form $\mathfrak{g}=H \oplus I$ with an abelian ideal $I$ the claim was established in part (b) of Lemma 2. This contradiction shows that our assumption was false; the claim is indeed true. 
To formulate our next theorem, we denote by $\operatorname{conj}(\mathfrak{g}, \sigma)$ the number of conjugacy classes of $\sigma$-invariant Cartan algebras under the action of the group $\left\langle e^{\text {ad } L}\right\rangle$ where $L=\{x \in \mathfrak{g} \mid \sigma x=x\}$.

Theorem 4. Let $\mathfrak{g}$ be a finite-dimensional Lie algebra over a field of characteristic zero, and let $R$ be the radical of $\mathfrak{g}$. Suppose that $R \neq\{0\}$ and that $\operatorname{conj}\left(\mathfrak{g} / R, \sigma_{R}\right)<\infty$. If $\sigma$ is an involution of $\mathfrak{g}$ and $\sigma_{R}$ is the involution of $\mathfrak{g} / R$ induced by $\sigma$, then $\operatorname{conj}(\mathfrak{g}, \sigma) \leq \operatorname{conj}\left(\mathfrak{g} / R, \sigma_{R}\right)$.

Proof. The proof is a modification of that of Theorem 3. Suppose that the claim is false, and pick a counterexample $(\mathfrak{g}, \sigma)$ of minimal dimension; then there are pairwise nonconjugate $\sigma$-invariant Cartan algebras $H_{1}, \ldots, H_{m}$ of $\mathfrak{g}$ where $m>\operatorname{conj}\left(\mathfrak{g} / R, \sigma_{R}\right)$. Choose a $\sigma$-invariant ideal $I \neq\{0\}$ of $\mathfrak{g}$ inside $R$, and let $\overline{\mathfrak{g}}:=\mathfrak{g} / I$ so that $\overline{\mathfrak{g}} / \bar{R}=(\mathfrak{g} / I) /(R / I) \cong \mathfrak{g} / R$. Since $\overline{\mathfrak{g}}$ has lower dimension than $\mathfrak{g}$ and hence is not a counterexample, we have $\operatorname{conj}(\overline{\mathfrak{g}}, \bar{\sigma}) \leq$ $\operatorname{conj}\left(\overline{\mathfrak{g}} / \bar{R}, \bar{\sigma}_{R}\right)=\operatorname{conj}\left(\mathfrak{g} / R, \sigma_{R}\right)<m ;$ hence the Cartan algebras $\overline{H_{1}}, \ldots, \overline{H_{m}}$ of $\overline{\mathfrak{g}}$ are no longer nonconjugate. Thus we can find indices $i \neq j$ and fixed points $\xi_{1}, \ldots, \xi_{n}$ of $\bar{\sigma}$ with

$$
\overline{H_{j}}=e^{\operatorname{ad} \xi_{1}} \cdots e^{\operatorname{ad} \xi_{n}} \overline{H_{i}} .
$$

Now if $\xi_{k}=a_{k}+I$ then also $\xi_{k}=\frac{1}{2}\left(a_{k}+\sigma a_{k}\right)+I$; hence each element $\xi_{k}$ can be written as $\xi_{k}=y_{k}+I$ where $y_{k}$ is a fixed point of $\sigma$. Then (6) becomes

$$
H_{j}+I \supseteq e^{\text {ad } y_{1}} \ldots e^{\text {ad } y_{n}} H_{i}=: H_{*} .
$$

Now $U:=H_{j}+I$ is a $\sigma$-invariant subalgebra of $\mathfrak{g}$ which is clearly solvable because $H_{j}$ is nilpotent and $I$ is a solvable ideal; moreover, $H_{*}$ and $H_{j}$ are $\sigma$-invariant Cartan algebras of $U$. But then Theorem 3 implies that there is an element $z \in U \subseteq \mathfrak{g}$ with $\sigma z=z$ such that

$$
H_{j}=e^{\text {ad } z} H_{*}=e^{\text {ad } z} e^{\text {ad } y_{1}} \ldots e^{\text {ad } y_{n}} H_{i} .
$$

This clearly contradicts our choice of $H_{1}, \ldots, H_{m}$ as being pairwise nonconjugate.

Combining Theorems 2 and 4, we obtain the following result.

Theorem 5. Let $\mathfrak{g}$ be a finite-dimensional real Lie algebra, and let $\sigma: \mathfrak{g} \rightarrow \mathfrak{g}$ be an involution with the corresponding symmetric decomposition $\mathfrak{g}=L \oplus Q$. Then the number of conjugacy classes of $\sigma$-invariant Cartan algebras under the action of $\left\langle e^{\text {ad } L}\right\rangle$ is finite.

Proof. We have $\operatorname{conj}(\mathfrak{g}, \sigma) \leq \operatorname{conj}\left(\mathfrak{g} / R, \sigma_{R}\right)$ by Theorem 4 and $\operatorname{conj}\left(\mathfrak{g} / R, \sigma_{R}\right)$ $<\infty$ by Theorem 2 because $\mathfrak{g} / R$ is semisimple.

It is often convenient to treat a question concerning the structure of Lie algebras for semisimple algebras and solvable algebras separately and then to obtain a result for arbitrary Lie algebras by using a suitable Levi decomposition. Hence we ask for the compatibility of $\sigma$-invariant Cartan algebras and $\sigma$-invariant Levi complements.

Let $R$ be the radical of a Lie algebra $\mathfrak{g}$. A Levi decomposition $\mathfrak{g}=R+S$ and a Cartan algebra $H$ of $\mathfrak{g}$ are called adapted if $H=(H \cap R)+(H \cap S)$. The following statements will be proved. Given a $\sigma$-invariant Cartan algebra $H$, there is a $\sigma$-invariant Levi complement $S$ such that $H=(H \cap R)+(H \cap S)$. 
Conversely, given a $\sigma$-invariant Levi complement $S$, there is a $\sigma$-invariant Cartan algebra $H$ such that $H=(H \cap R)+(H \cap S)$. Of course, the special choice $\sigma:=\mathrm{id}_{\mathfrak{g}}$ gives the well-known adaptability claim in the case where no involution is considered.

Theorem 6. Let $\mathfrak{g}$ be a finite-dimensional real Lie algebra with radical $R$, and let $\sigma: \mathfrak{g} \rightarrow \mathfrak{g}$ be an involution.

(a) Suppose $S$ is a $\sigma$-invariant Levi complement with a $\sigma$-invariant Cartan algebra $H_{S}$. (The existence of $H_{S}$ was proved in Theorem 1.) Then there is a $\sigma$-invariant subalgebra $H_{R}$ of $R$ such that $H:=H_{R}+H_{S}$ is a Cartan algebra of $R$. Obviously, $H$ is $\sigma$-invariant and adapted to the decomposition $\mathfrak{g}=R+S$.

(b) Suppose conversely that $H$ is a $\sigma$-invariant Cartan algebra of $\mathfrak{g}$. Then there exist a $\sigma$-invariant Levi complement $S$, a $\sigma$-invariant Cartan algebra $H_{S}$ of $S$, and a $\sigma$-invariant subalgebra $H_{R}$ of $R$ such that $H=H_{R}+H_{S}$.

Proof. (a) Since $S$ is semisimple, the Cartan algebra $H_{S}$ of $S$ is abelian, and for any finite-dimensional representation $\phi: S \rightarrow \operatorname{gl}(V)$ all operators $\phi(h)$ $\left(h \in H_{S}\right)$ are semisimple; in particular, all operators $\operatorname{ad}(h) \quad\left(h \in H_{S}\right)$ are semisimple. This implies that the Cartan algebras of $\mathfrak{g}$ which contain $H_{S}$ are exactly the Cartan algebras of

$$
C:=Z_{\mathfrak{g}}\left(H_{S}\right)=\left\{x \in \mathfrak{g} \mid\left[x, H_{S}\right]=\{0\}\right\}
$$

(see [1, VII.2.3, Proposition 10, p. 24]). Now $C$ is $\sigma$-invariant, since $H_{S}$ is. By Theorem 1, there is a $\sigma$-invariant Cartan algebra $H$ of $C$. By what was said before, $H$ is a Cartan algebra of $\mathfrak{g}$ which contains $H_{S}$. Now $\bar{H}:=\left(H+H_{S}\right) / H_{S}$ is a Cartan algebra of $\bar{C}:=C / H_{S}$. We claim that the mapping

$$
f: \begin{aligned}
R \cap C & \rightarrow \bar{C} \\
r & \mapsto r+H_{S}
\end{aligned}
$$

is onto. Indeed, let $c \in C$ be written as $c=r+s$ with $r \in R$ and $s \in S$. Then $\left[r+s, H_{S}\right]=\{0\}$ implies $\left[r, H_{S}\right]=\{0\}$ and $\left[s, H_{S}\right]=\{0\}$, whence $r \in C$ and $s \in H_{S}$ (because $H_{S}$ is maximal abelian in $S$ ). But this shows that $c \in r+H_{S}$ with $r \in R \cap C$.

Note that $f^{-1}(\bar{H})$ is a $\sigma$-invariant subalgebra of $R \cap C$ and, hence, possesses a $\sigma$-invariant Cartan algebra $H_{R}$ by Theorem 1. We claim that $H_{R}$ is a Cartan algebra of all of $R \cap C$, not only of $f^{-1}(\bar{H})$. Obviously, $H_{R}$ is nilpotent. Let $x \in N_{R \cap C}\left(H_{R}\right)$. Then $f(x) \in N_{f(R \cap C)} f\left(H_{R}\right)=N_{\bar{C}}(\bar{H})=\bar{H}$ so that $x \in f^{-1}(\bar{H})$. This implies $x \in N_{f^{-1}(\bar{H})}\left(H_{R}\right)=H_{R}$. Since $f$ is onto, the image $f\left(H_{R}\right)$ is a Cartan algebra of $\bar{C}$. But obviously $f\left(H_{R}\right) \subseteq \bar{H}$, and $\bar{H}$ is also a Cartan algebra of $\bar{C}$. This gives $f\left(H_{R}\right)=\bar{H}$, i.e., $\left(H_{R}+H_{S}\right) / H_{S}=$ $\left(H+H_{S}\right) / H_{S}$. Consequently, $H_{R}+H_{S}=H+H_{S}=H$ where we used the fact that $H \supseteq H_{S}$.

(b) Choose any $\sigma$-invariant Levi complement $[S]$; this is possible by [4, Volume II, pp. 327-329]. Then

$$
H_{[S]}:=(H+R) \cap[S]
$$

is a $\sigma$-invariant Cartan algebra of $[S]$. (The $\sigma$-invariance is clear, and $H_{[S]}$ is a Cartan algebra as the homomorphic image of the Cartan algebra $H$ of $\mathfrak{g}$ under 
the homomorphism $\mathfrak{g}=R+[S] \rightarrow[S]$.) By part (a), there is a $\sigma$-invariant subalgebra $H_{[R]}$ of $R$ such that

$$
[H]:=H_{[R]}+H_{[S]}
$$

is a Cartan algebra of $\mathfrak{g}$. Now

$$
H+R=(H+R) \cap S+R=H_{[S]}+R \subseteq[H]+R \subseteq H_{[S]}+R \subseteq H+R
$$

so that $H+R=[H]+R=H_{[S]}+R=: A$. The fact that $H_{[S]}$ is abelian implies $[A, A] \subseteq R$ so that $A$ is a solvable $\sigma$-invariant algebra. Moreover, $H$ and $[H]$ are $\sigma$-invariant Cartan algebras of $\mathfrak{g}$ contained in $A$ and, hence, $\left.\sigma\right|_{A}$-invariant Cartan algebras of $A$. By Theorem 3, there is an element $x \in A$ with $\sigma x=x$ such that $\gamma:=e^{\text {ad } x}$ satisfies $H=\gamma[H]$. Let

$$
S:=\gamma[S], \quad H_{S}:=\gamma H_{[S]}, \quad H_{R}:=\gamma H_{[R]} .
$$

Clearly, $S$ is a $\sigma$-invariant Levi complement, $H_{S}$ is a $\sigma$-invariant Cartan algebra of $S$, and $H_{R}$ is a $\sigma$-invariant subalgebra of $R$; the $\sigma$-invariance is guaranteed by the special form of the automorphism $\gamma$. Furthermore,

$$
H=\gamma[H]=\gamma\left(H_{[R]}+H_{[S]}\right)=H_{R}+H_{S} .
$$

\section{REFERENCES}

1. Nicolas Bourbaki, Groupes et algèbres de Lie, Chapitres 7 et 8, Hermann, Paris, 1975.

2. Sigurdur Helgason, Differential geometry, Lie groups and symmetric spaces, Academic Press, New York, San Francisco, and London, 1978.

3. Joachim Hilgert, Karl Heinrich Hofmann, and Jimmie D. Lawson, Lie groups, convex cones, and semigroups, Oxford Univ. Press, Oxford, 1988.

4. Shoshichi Kobayashi and Katsumi Nomizu, Foundations of differential geometry. I, II, Wiley (Interscience), New York, 1963/1969.

5. Henrik Schlichtkrull, Hyperfunctions and harmonic analysis on symmetric spaces, Birkhäuser, Boston, Basel and Stuttgart, 1984.

6. Garth Warner, Harmonic analysis on semi-simple Lie groups. I, Springer, Berlin, Heidelberg, and New York, 1972.

Department of Mathematics, Louisiana State University, Baton Rouge, Louisiana 70803

Current address: Robert-Koch-Straße 12, D-64521 Groß-Gerau, Germany

E-mail address: KSPINDLE\%ESOC. BITNETQvm.gmd.de 